\title{
Sazonalidade da fauna de metazoários de Pygocentrus nattereri (Kner, 1858) no lago Piranha (Amazonas, Brasil) e a avaliação de seu potencial como indicadora da saúde do ambiente
}

\author{
José Francalino Vital ${ }^{1,2}$, Angela Maria Bezerra Varella ${ }^{1}$, \\ Daniel Brito Porto ${ }^{1}$ \& José Celso de Oliveira Malta ${ }^{1}$ \\ ${ }^{1}$ Laboratório de Parasitologia e Patologia de Peixes, Coordenação de Pesquisas em Biologia Aquática, \\ Instituto Nacional de Pesquisas da Amazônia - INPA. Av. André Araújo, 2936, Aleixo, \\ CEP 69060-001, Manaus, AM, Brasil \\ ${ }^{2}$ Autor para correspondência: José Francalino Vital, e-mail: jfvital@yahoo.com.br
}

VITAL, J.F., VARELLA, A.M.B., PORTO, D.B. \& MALTA, J.C.O. Seasonality of the metazoan fauna of Pygocentrus nattereri (Kner, 1858) in Piranha Lake, (Amazonas, Brazil), and evaluation of its potential as an indicator of environmental health. Biota Neotrop. 11(1): http://www.biotaneotropica.org.br/v11n1/en/ abstract?article+bn03111012011.

\begin{abstract}
The metazoan parasitic fauna of Pygocentrus nattereri collected in different phases of hydrologic cycle of Piranha lake was studied during the year of 2007. The study area is located in gas pipeline Coari-Manaus construction zone, near the municipality of Manacapuru-Am. The relationship between condition factor and parasite occurrence and the viability of use the parasitic fauna as an environmental bio-indicator were verified. The following Monogenoidea were collected: Amphithecium microphalum, Amphitecium brachycirrum, Amphitecium calycinum, Amphithecium catalaoensis, Amphithecium junki, Pithanothecium amazonensis e Rhinoxenus piranhus, as well as the nematode Procamallanus inopinatus, the copepod Miracetyma sp. and the isopod Anphira branchialis. There was not significant correlation between the parasite occurrence and the condition factor. There were significant variances in the parasitic index according to the different periods of hydrologic cycle and $P$. nattereri has achieved the conditions described in the literature to be select a fish-host and his parasite fauna as biomonitor.
\end{abstract}

Keywords: parasitism, piranha, Amazon, enviromental biomonitoring, fish parasites.

VITAL, J.F., VARELLA, A.M.B., PORTO, D.B. \& MALTA, J.C.O. Sazonalidade da fauna de metazoários de Pygocentrus nattereri (Kner, 1858) no lago Piranha (Amazonas, Brasil), e a avaliação de seu potencial como indicadora da saúde do ambiente. Biota Neotrop. 11(1): http://www.biotaneotropica.org.br/v11n1/pt/ abstract?article+bn03111012011.

Resumo: Foi estudada a fauna de metazoários parasitos de Pygocentrus nattereri capturadas no lago Piranha, durante as fases do ciclo hidrológico do ano de 2007. Foi verificada a relação entre o fator de condição e a ocorrência de parasitos, e também a viabilidade da utilização da fauna parasitária de $P$. nattereri como bioindicadora da saúde do ambiente. Foram coletados monogenóideos das espécies Amphithecium microphalum, Amphitecium brachycirrum, Amphitecium calycinum, Amphithecium catalaoensis, Amphithecium junki, Pithanothecium amazonensis e Rhinoxenus piranhus, o nematóide Procamallanus inopinatus, copépodos da espécie Miracetyma sp. e o isópoda Anphira branchialis. Não houve correlação significativa entre a ocorrência dos parasitos com o fator de condição dos peixes. Houve variações significativas entre os índices parasitários nos diferentes períodos do ciclo hidrológico e $P$. nattereri satisfez os requisitos descritos na literatura para selecionar um peixe hospedeiro e sua parasitofauna como bioindicadora.

Palavras-chave: parasitismo, piranha, Amazônia, monitoramento ambiental, parasitos de peixes. 


\section{Introdução}

O parasitismo pode ser empregado como monitor altamente sensível na detecção de mudanças que ocorrem em habitats degradados, podendo ser indicador de contaminações ambientais e da saúde ambiental (Overstreet 1997).

As infestações parasitárias e sua relação com mudanças no ambiente devido à presença de materiais poluentes têm sido frequentemente abordadas como alternativa para monitoramento ambiental, pelo efeito direto nas populações de parasitos ou pela avaliação dos hospedeiros paratênicos, intermediários e finais. A análise de populações e da estrutura de comunidades de parasitos pode ser vantajosa se comparada com os estudos usando organismos de vida livre, por causa da sobreposição dos efeitos dos parâmetros ambientais em diferentes níveis tróficos, entretanto é preciso diferenciar as variações naturais nas populações de parasitos daquelas que são motivadas por impactos ambientais antrópicos (Sures 2004).

Nos lagos amazônicos o pulso de inundação é responsável pela formação de uma área de transição aquática-terrestre, que muda sazonalmente pela ação do somatório das águas das chuvas de toda a área drenada e do degelo que ocorre na região andina durante o verão. Ao longo do ano ocorrem profundas mudanças, caracterizando um ambiente bastante dinâmico (Junk et al. 1989).

Mudanças sazonais no ambiente podem causar modificações na relação parasito-hospedeiro. A presença ou abundância de parasitos é diretamente influenciada tanto pelo ambiente no interior do hospedeiro como pela condição do ecossistema (Kadlec et al. 2003).

As áreas alagáveis apresentam alterações na dinâmica populacional da fauna autóctone, principalmente nas faunas malacológica e ictiológica, tendo reflexos diretos ou indiretos na estrutura e composição das espécies de parasitos de peixes e conseqüentemente em seu ciclo de vida (Pavanelli et al. 1997).

A composição da comunidade parasitária depende de vários fatores relacionados ao ambiente (baixa qualidade da água, alterações do pH, concentração de amônia, disponibilidade de oxigênio dissolvido, variações na temperatura, nível da água e efeitos da sazonalidade), ao hospedeiro (habitat, comportamento alimentar, fisiologia, idade e sexo) e ao parasito (disponibilidade de larvas infectantes, de hospedeiros individuais, da resposta imune do hospedeiro ao estabelecimento da larva e da mortalidade natural dos parasitos) (Takemoto et al. 2004).

Pygocentrus nattereri (Kner 1858), popularmente conhecida como piranha vermelha, pertence à família Characidae e à subfamília Serrasalminae, é um peixe piscívoro e um dos principais predadores dos lagos de várzea amazônicos (Fink 1993). P. nattereri alimenta-se arrancando pedaços de suas presas, possui desova parcelada, reproduz no início da enchente e a primeira maturação sexual ocorre em peixes com $13 \mathrm{~cm}$ nos machos e $15 \mathrm{~cm}$ nas fêmeas (Santos et al. 2006).

Devido à crescente atividade da indústria petrolífera na região amazônica torna-se necessária a análise das características ecológicas de seus ambientes antes de qualquer impacto antrópico. $\mathrm{O}$ conhecimento da dinâmica ecológica natural desses ambientes é primordial para a seleção de espécies indicadoras da qualidade ambiental e o desenvolvimento de mecanismos de controle e avaliação das alterações que possam ocorrer. O conhecimento da fauna parasitológica de $P$. nattereri do lago Piranha é uma ferramenta adicional, que poderá ser usada no monitoramento das condições desse ambiente.

\section{Material e Métodos}

\section{1. Área de estudo e coleta dos hospedeiros e parasitos}

Os peixes foram capturados no lago Piranha, um lago de várzea localizado à margem esquerda do Rio Solimões, a 110 km de Manaus e $25 \mathrm{~km}$ de Manacapuru ( $3^{\circ} 17^{\prime} 40^{\prime \prime} \mathrm{S}$ e $60^{\circ} 35^{\prime} 32^{\prime}$ ' W), região de construção do gasoduto Coari-Manaus. O ecossistema local é inteiramente de várzea com profundas mudanças sazonais no nível das águas, influenciadas pelos fenômenos de enchente e vazante do Rio Solimões.

Quatro coletas foram realizadas durante os meses de abril (enchente), junho (cheia), setembro (vazante) e novembro (seca) de 2007. As coletas foram realizadas com redes de espera de tamanhos de malhas variando de 20 a $90 \mathrm{~mm}$ entre nós adjacentes, dispostas aleatoriamente no lago. Após a captura os peixes foram armazenados em caixas de isopor entre camadas de gelo e em menos de 24 horas transportados para o Laboratório de Parasitologia e Patologia de Peixes (LPP-INPA). Posteriormente foram pesados, medidos e necropsiados. Os parasitos encontrados foram fixados segundo a metodologia descrita por Amato et al. (1991). Os procedimentos para identificação dos parasitos (clarificação, coloração e montagem em lâminas) foram realizados de acordo com a metodologia específica para cada grupo (Eiras et al. 2006, Amato et al. 1991).

Os índices parasitários das populações de parasitos foram feitos segundo Bush et al. (1997) e Serra-Freire (2002).

\section{Fator de condição dos hospedeiros}

Com os logaritmos dos valores de comprimento padrão (Ls) e de peso total (Wt) de cada peixe, foi ajustada a curva da relação $\mathrm{Wt} / \mathrm{Ls}$ e foram estimados os valores dos coeficientes de regressão $a$ e $b$. Os valores de $a$ e $b$ foram utilizados nas estimativas dos valores teoricamente esperados de peso corporal (We) pela utilização da equação $W e=a . L t b$. Foi então calculado o fator de condição relativo $(\mathrm{Kn})$ que corresponde ao quociente entre o peso observado e o peso teoricamente esperado para um determinado comprimento $(\mathrm{Kn}=\mathrm{Wt} / \mathrm{We})($ Le Cren 1951).

\section{Análises estatísticas e avaliação do potencial da parasitofauna como bioindicadora}

As análises estatísticas foram realizadas segundo Zar (1999) e Serra-Freire (2002). O teste de análise de variância para dados não paramétricos Kruskal Wallis e o teste de comparações múltiplas de Dunn foram utilizados para comparar as prevalências e as intensidades médias de infecção entre os períodos do ciclo hidrológico (Ayres et al. 2003).

A existência de correlações entre a abundância das espécies de parasitos com o fator de condição relativo dos hospedeiros foram testadas por meio do coeficiente de correlação de Pearson " $r$ ". O potencial da parasitofauna de $P$. nattereri como bioindicadora foi avaliado conforme os itens propostos por Overstreet (1997) e Sures (2004).

\section{Resultados}

Foram examinados 80 exemplares de $P$. nattereri, 20 capturados em cada estação do ciclo hidrológico. Os indivíduos pesavam em média 72,09 g $\pm 41,11$ e o comprimento padrão médio foi de $11,17 \mathrm{~cm} \pm 1,80$, sendo que todos os peixes estavam parasitados por pelo menos um grupo de metazoários.

Foram encontradas sete espécies de Monogenoidea, da família Dactylogyridae parasitando P. nattereri: Amphithecium microphalum Kritsky, Boeger \& Jégu, 1997; Amphithecium brachycirrum Boeger \& Kritsky, 1988; Amphithecium calycinum Boeger \& Kritsky, 1988; Amphithecium catalaoensis Boeger \& Kritsky, 1988; Amphithecium junki Boeger \& Kritsky, 1988; Pithanothecium amazonensis (Mizelle \& Price, 1965); e Rhinoxenus piranhus Kritsky et al.1988; o nematóide Procamallanus inopinatus (Pereira, 1985); copépodos da espécie 
Tabela 1. Táxons de metazoários parasitos encontrados em Pygocentrus nattereri coletados nos meses de abril (enchente), junho (cheia), setembro (vazante) e novembro (seca) de 2007 no lago Piranha.

Table 1. Taxa of metazoan parasites found in Pygocentrus nattereri collected in the months of April (flood), June (high water), September (dry seanson), and November (low water) of 2007 in the Piranha Lake.

\begin{tabular}{|c|c|c|c|c|c|c|c|}
\hline Parasitos & Estações & PP/PE & $\mathbf{T P}$ & $\mathbf{P \%}$ & IM & AI & $\mathbf{A M}$ \\
\hline \multirow[t]{5}{*}{ Monogenoidea (B) } & enchente & $20 / 20$ & 1951 & 100 & 92,7 & $10-307$ & 92,7 \\
\hline & cheia & $20 / 20$ & 1788 & 100 & 89,4 & $11-259$ & 89,4 \\
\hline & vazante & $20 / 20$ & 3435 & 100 & 171,75 & $12-370$ & 171,75 \\
\hline & seca & $20 / 20$ & 9786 & 100 & 489,3 & $50-1111$ & 489,3 \\
\hline & Total & $80 / 80$ & 16960 & 100 & 210,78 & $10-1111$ & 210,78 \\
\hline \multirow[t]{5}{*}{ Procamallanus inopinatus (I) } & enchente & $18 / 20$ & 125 & 90 & 6,94 & $1-46$ & 6,25 \\
\hline & cheia & $14 / 20$ & 37 & 70 & 2,64 & $1-6$ & 1,85 \\
\hline & vazante & $08 / 20$ & 13 & 40 & 1,62 & $1-3$ & 0,65 \\
\hline & seca & $08 / 20$ & 8 & 45 & 1,11 & $1-1$ & 0,4 \\
\hline & Total & $48 / 80$ & 183 & 60 & 3,81 & $1-46$ & 2,28 \\
\hline \multirow[t]{5}{*}{ Miracetyma sp. (B) } & enchente & $10 / 20$ & 16 & 50 & 1,8 & $1-4$ & 0,8 \\
\hline & cheia & $03 / 20$ & 4 & 15 & 2,0 & $1-3$ & 0,2 \\
\hline & vazante & $03 / 20$ & 19 & 15 & 8,0 & $1-18$ & 0,95 \\
\hline & seca & $04 / 20$ & 27 & 20 & 9,0 & $1-16$ & 1,35 \\
\hline & Total & $20 / 80$ & 66 & 25 & 3,3 & $1-16$ & $\mathbf{0 , 8 2}$ \\
\hline
\end{tabular}

$\mathrm{B}=$ brânquias; $\mathrm{I}=$ intestino; $\mathrm{PP}=$ peixes parasitados; $\mathrm{PE}=$ peixes examinados; $\mathrm{TP}=$ Total de parasitos; $\mathrm{P} \%=$ Prevalência; $\mathrm{IM}=$ Intensidade média; $\mathrm{AI}=$ amplitude da intensidade; $\mathrm{AM}=$ abundância média.

Miracetyma sp. e o isópoda Anphira branchialis Thatcher, 1993. Os índices parasitários dos peixes estão registrados na Tabela 1.

Foram coletados 16960 exemplares de Monogenoidea nos arcos branquiais dos peixes. A prevalência foi de $100 \%$ e a intensidade média aumentou ao longo do ciclo hidrológico (Figura 1). A maior intensidade média ocorreu na seca (novembro) e houve diferença significativa entre os períodos de coleta $(\mathrm{p}<0,0001)$. Foi observado uma correlação positiva e significativa entre o fator de condição e a abundância de Monogenoidea, $(r=0,228$ e p = 0,042) (Figura 2).

Um total de 183 indivíduos de $P$. inopinatus foram coletados no intestino de $P$. nattereri, com prevalência de $25 \%$. Houve diferença significativa da prevalência e da intensidade média entre os períodos de coleta $(p=0,0001)$ com a diminuição de ambas ao longo do ciclo hidrológico (Figuras 3 e 4). Os períodos de enchente e cheia (abril e junho) foram os que apresentaram maior diferença em relação aos outros períodos de coleta. $\mathrm{O}$ fator de condição não apresentou correlação com a ocorrência de nematóides $(r=0,0770$ e $p=0,500)$.

Um total de 66 Miracetyma sp. foram coletados nos arcos branquiais dos peixes analisados. Embora a prevalência tenha diminuído (Figura 5), a intensidade média aumentou ao longo do ciclo hidrológico (Figura 6), mas os índices de Miracetyma sp. não apresentaram diferença significativa entre os períodos de coleta $(\mathrm{p}=0,1384)$.

Os exemplares de A. branchialis foram coletados nos arcos branquiais em apenas duas fases do ciclo hidrológico (vazante e seca). Estes exemplares foram encontrados parasitando as brânquias dos peixes apenas em um hospedeiro da coleta realizada na vazante $\mathrm{e}$ dois hospedeiros da coleta realizada na seca. Para estes parasitos não foi possível calcular todos os índices, pelo fato de terem ocorrido em apenas três hospedeiros analisados.

\section{Discussão}

As variações nas infrapopulações de parasitos podem ocorrer devido às mudanças naturais do ambiente, ou podem também variar como resultado de efeitos tóxicos diretos, nos parasitos ou seus hospedeiros, e ainda efeitos ambientais indiretos em populações de hospedeiros intermediários (Poulin 1992).

Segundo Mackenzie et al. (1995) o aumento das infrapopulações de espécies parasitos com ciclo de vida direto, usualmente protozoários e monogenéticos, pode ser devido a um efeito geralmente atribuído ao comprometimento das respostas imunes do hospedeiro.

Neste trabalho as espécies de Monogenoidea foram as que apresentaram os maiores índices parasitários e o aumento nas infestações foi marcante no período seco. Como nesta estação os lagos de várzea secam rapidamente há uma fuga frenética dos organismos aquáticos para o leito do rio ou lagos, para poderem sobreviver. Muitos não conseguem, ou morrem por falta de água ou são facilmente predados em rasas poças de água. Essa pressão ambiental gera um grande estresse nos peixes, comprometendo as respostas imunes e fazendo que os índices parasitários sejam maiores, corroborando com o que relatou Mackenzie et al. (1995).

Procamallanus peraccurattus Pinto, Fábio, Noronha \& Rolas, 1974 parasito de Geophagus brasiliensis Quoy \& Gaimard, 1824 capturados nos reservatórios de Jaguari e Juqueri, na região leste do Estado de São Paulo, apresentaram os maiores índices parasitários na estação de seca (Madi 2005). Várias espécies de Procamallanus parasitos de Clarias sp. na Malásia e Egito, também apresentaram os maiores índices parasitários na estação seca (Furtado \& Tan 1973).

Nesse trabalho os maiores índices parasitários de $P$. inopinatus ocorreram nas estações de enchente e cheia, resultados diferentes dos encontrados por Furtado \& Tan (1973). Isto deve-se porque nas estações de enchente e cheia na Amazônia as condições ambientais são as melhores para vários organismos aquáticos, assim há uma grande quantidade de indivíduos viabilizando o encontro das formas larvais infectantes com seus hospedeiros, permitindo a complementação do ciclo de vida das diversas espécies.

Três espécies de Copepoda já foram referidas parasitando P. nattereri: Miracetyma piraya Malta, 1994 coletada no Rio Mamoré (Malta 1994), Ergasilus yumaricus Malta \& Varella, 1996 coletada nos rios Guaporé, Mamoré, Jiparaná e Pacaás-Novos no Estado de Rondônia (Malta \& Varella 1996), ambas parasitando os filamentos 


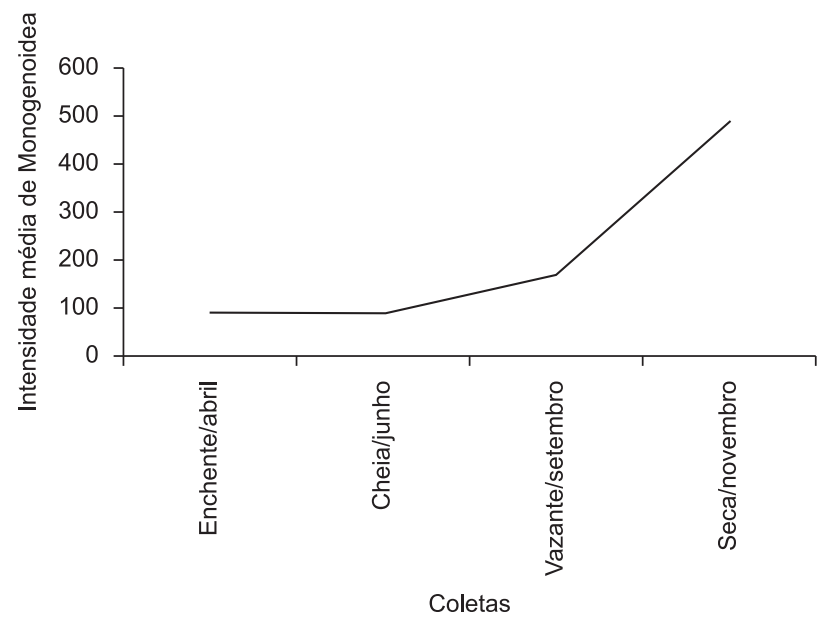

Figura 1. Variação da intensidade média das espécies de Monogenoidea, parasitos de Pygocentrus nattereri, durante um ciclo hidrológico anual do lago Piranha.

Figure 1. Variation in the mean intensity of Monogenoidea parasites of Pygocentrus nattereri during an annual hydrological cycle of Piranha Lake.

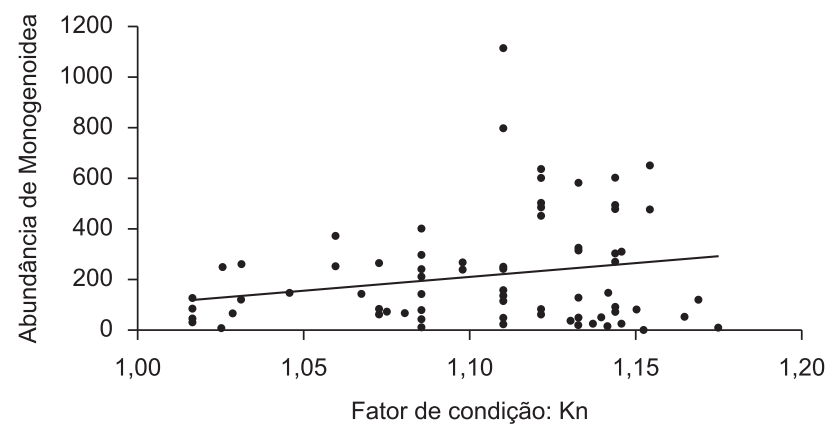

Figura 2. Teste de correlação linear de Pearson entre o fator de condição e a abundância das espécies de Monogenoidea em Pygocentrus nattereri.

Figure 2. Pearsons correlation linear test between condition factor and the abundance of Monogenoidea, parasites of Pygocentrus nattereri, during an annual hydrological cycle of Piranha Lake.

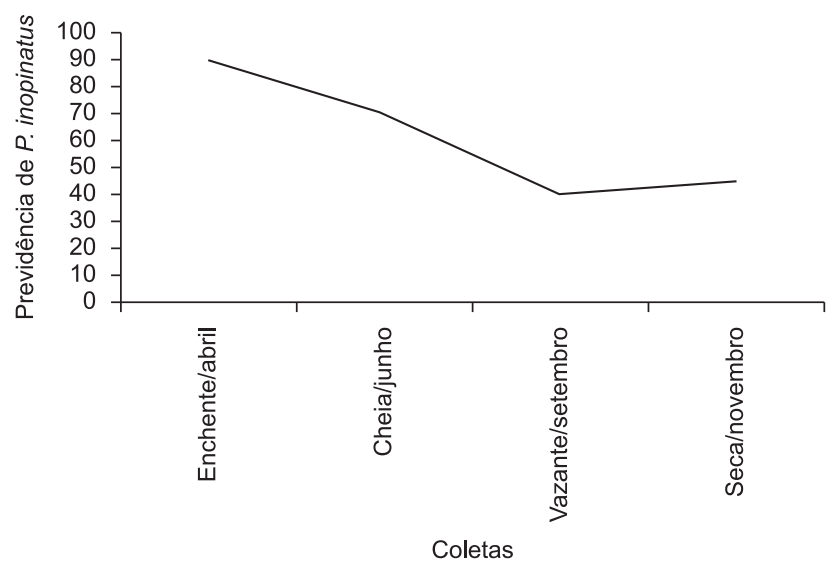

Figura 3. Variação da prevalência de Procamallanus inopinatus, parasito de Pygocentrus nattereri, durante um ciclo hidrológico anual do lago Piranha.

Figure 3. Variation in the prevalence of Procamallanus inopinatus, parasite of Pygocentrus nattereri, during an annual hydrological cycle of Piranha Lake. branquiais, e Rhinergasilus piranhus Boeger \& Thatcher, 1988, parasito das fossas nasais, coletada no Rio Solimões próximo à Manaus (Boeger \& Thatcher 1988).

Nesse trabalho foi encontrada somente uma espécie de Copepoda, Miracetyma sp. parasitando os filamentos branquiais e sua prevalência foi de $25 \%$, três vezes maior que a encontrada por Malta (8\%). Já a intensidade média foi de 3,3 enquanto Malta registrou 8,0. Os índices foram discrepantes possivelmente pelas diferenças nas amostragens e nas áreas de coleta.

No Rio Araguaia, tributário do Rio Tocantins, no Estado de Goiás foram encontradas três espécies de Isopoda parasitando $P$. nattereri, Braga patagonica Schiödte \& Meinert, 1884; Anphira branchialis Thatcher, 1993 e Asotana sp. (Carvalho et al. 2004). No presente trabalho foi encontrada Anphira branchialis parasitando $P$. nattereri. Assim como no trabalho de Carvalho et al. (2004) todos foram encontrados na estação seca parasitando os arcos branquiais. A prevalência foi muito baixa comparada com a encontrada pelos autores acima. Essas diferenças tanto na fauna quanto nos índices parasitários podem ser atribuídas às condições diferentes das localidades estudadas, Rio Araguaia e Rio Solimões.

Os efeitos que agentes patogênicos causam em seus hospedeiros necessitam de parâmetros para serem avaliados. Uma análise inicial consiste em definir um "normal" ou controle com o qual possamos comparar os indivíduos parasitados (Chubb 1973). Para os peixes, o fator de condição, que é uma medida ou um indicador quantitativo do bem-estar, pode servir como ferramenta de estudo das relações entre o estado de saúde e o parasitismo (Brasil-Sato 1999, Lizama 2003).

O fator de condição e a presença ou abundância de determinadas espécies de parasitos são variáveis relacionadas (Lemly 1980, Isaac et al. 2004). Porém, frequientemente, em condições naturais os peixes são infectados por muitas espécies que coexistem e demonstram inter-relações, ou seja, cada indivíduo hospedeiro abriga uma infracomunidade de parasitos (Bush et al. 1997). Assim, há de se considerar também o efeito desses conjuntos de espécies sobre a condição dos hospedeiros.

Nesse trabalho com $P$. nattereri foi observada uma correlação positiva e significativa entre o fator de condição e a abundância de Monogenoidea, ao contrário de Guidelli (2006) que trabalhando com Leporinus elongatus Valenciennes, 1849; L. friderici (Bloch, 1794); L. lacustris Campos, 1945 e L. obtusidens (Valenciennes, 1836), da planície de inundação do alto Rio Paraná, encontrou correlações negativas e significativas, segundo a autora isto ocorreu possivelmente porque os indivíduos de $P$. nattereri com melhores condições tiveram melhores possibilidades de reagir eficazmente à infestação pelas espécies de Monogenoidea e possivelmente seu sistema imune estava mais apto a combater as reações ao parasitismo.

A relação entre a carga parasitária de Rondonia rondoni Travassos, 1920 (Nematoda, Atractidae) e o fator de condição de Pterodoras granulosus Valenciennes, 1833 foram estudados no Rio Paraná. A intensidade média de parasitismo e a prevalência de $R$. rondoni aumentaram com o acréscimo do comprimento do hospedeiro, possivelmente em função da maior ingestão de alimento pelos hospedeiros maiores e pelo processo de acúmulo progressivo dos parasitos. Porém, a intensidade de infecção não afetou o fator de condição dos hospedeiros (Dias et al. 2004).

Resultados semelhantes foram encontrados por Campos (2006) com Pseudoplatystoma fasciatum (Spix \& Agassiz, 1829) do Rio Aquidauana, Pantanal Mato-grossense, parasitados por seis espécies de Cestoda no intestino. Essa endofauna não apresentou correlação significativa entre fator de condição e abundância parasitária.

Nesse trabalho não houve correlação significativa entre o fator de condição e a abundância parasitária da endofauna, representada 


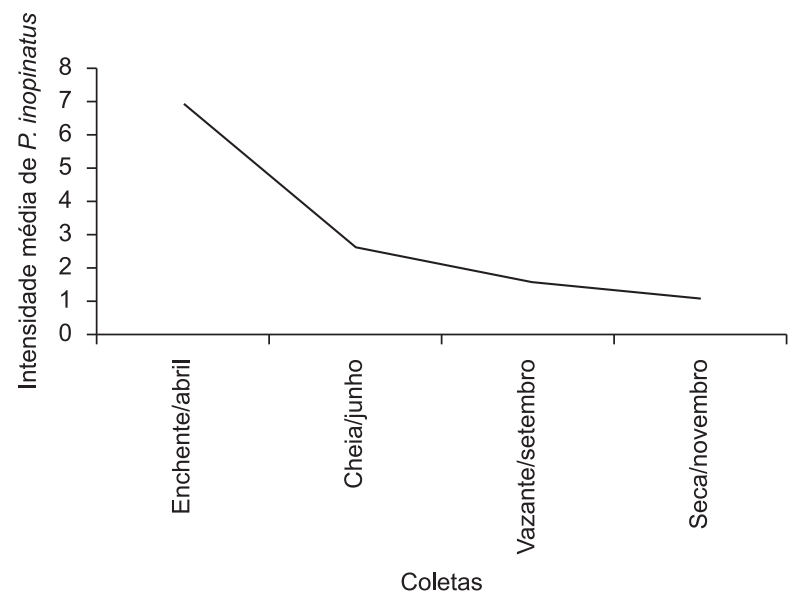

Figura 4. Variação da intensidade média de Procamallanus inopinatus, parasito de Pygocentrus nattereri, durante um ciclo hidrológico anual do lago Piranha.

Figura 4. Variation in the mean intensity of Procamallanus inopinatus, parasite of Pygocentrus nattereri, during an annual hydrological cycle of Piranha Lake.

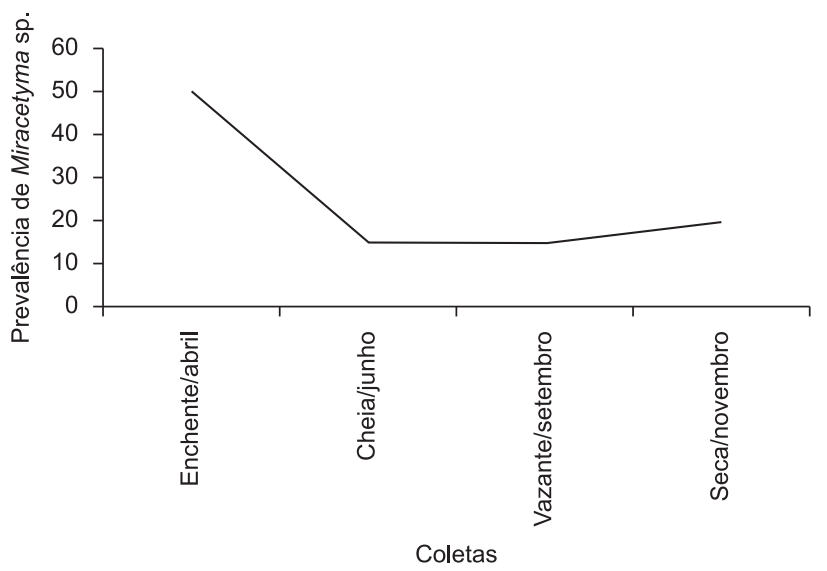

Figura 5. Variação da prevalência de Miracetyma sp., parasito de Pygocentrus nattereri, durante um ciclo hidrológico anual do lago Piranha.

Figure 5. Variation in the prevalence of Miracetyma sp., parasite of Pygocentrus nattereri during an annual hydrological cycle of Piranha Lake.

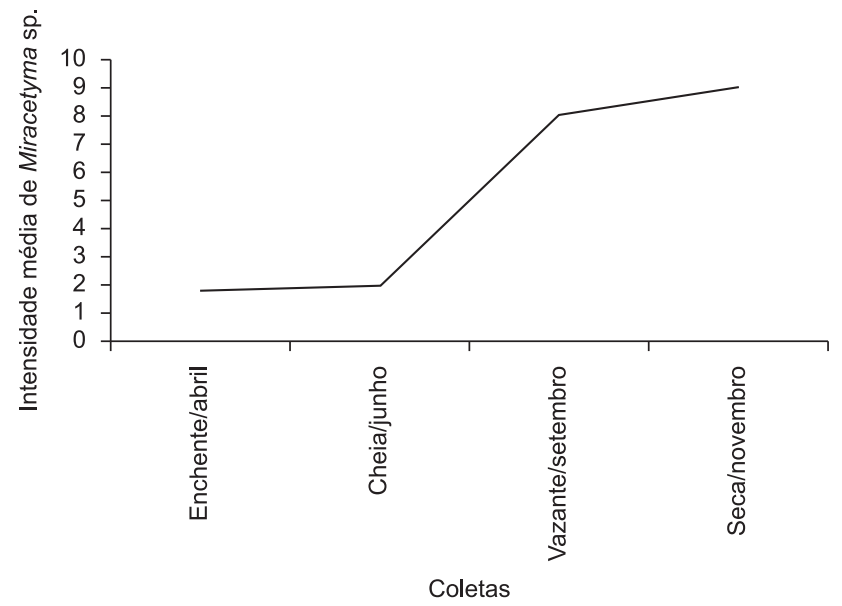

Figura 6. Variação da intensidade média de Miracetyma sp., parasito de Pygocentrus nattereri durante um ciclo hidrológico anual do lago Piranha.

Figure 6. Variation in the mean intensity of Miracetyma sp., parasite of Pygocentrus nattereri during an annual hydrological cycle of Piranha Lake. por $P$. inopinatus. Resultado semelhante ao que encontrou Campos (2006) e Dias et al. (2004) em seus estudos, e diferentes ao de Guidelli (2006) que encontrou uma covariação positiva. Esse fato ocorreu provavelmente pelo número reduzido de $P$. inopinatus encontrados por hospedeiro

$P$. nattereri apresenta-se como uma espécie que tem as características exigidas para que sua parasitofauna seja usada como bioindicadora: é abundante nos lagos de várzea da Amazônia; uma das espécies mais fáceis de ser capturada; é fácil de ser identificada; tem ampla distribuição na Amazônia e no Brasil; vive em áreas restritas, os lagos de várzea; é hospedeira de um número elevado de parasitos, nesse trabalho nove espécies. Apenas uma das exigência não é atendida, ter tamanho pequeno, pois seu porte é médio. No entanto a coleta e identificação de seus parasitos pode ser feita em pouco tempo, portanto esta exigência não a elimina de ser considerada um peixe-modelo para o uso de sua parasitofauna como bioindicadora.

Algumas populações de parasitos podem responder positivamente a impactos antrópicos enquanto outras podem responder negativamente. Os Monogenoidea, que possuem ciclo de vida monoxeno, via de regra aumentam suas infestações em resposta a impactos antrópicos, já os parasitos de ciclo de vida heteroxeno podem ter suas infestações reduzidas devido à diminuição das populações dos seus hospedeiros intermediários (Marcogliese \& Cone 1997). No presente estudo verificamos que $P$. nattereri possui parasitos tanto de ciclo de vida heteroxeno ( $P$. inopinatus) como monoxeno (todas as demais espécies encontradas), um fator importante que aponta o potencial para o desenvolvimento de uma ferramenta de biomonitoramento, por meio do acompanhamento dessas populações de parasitos.

Valores da prevalência e da intensidade média de um específico parasito, em diferentes localidades demonstram a diferente natureza dos habitats, em termos de associação de invertebrados de vida livre e vertebrados hospedeiros. A teia de animais de uma comunidade que co-ocorre com os peixes em habitats relativamente saudáveis nos dá um indicativo da biodiversidade (Overstreet 1997).

A fauna de parasitos e os valores dos índices parasitários foram registrados e conforme Overstreet 1997, fica caracterizado a natureza dos habitats em termos de associação de invertebrados de vida livre e parasitos com $P$. nattereri no lago Piranha. Os dados obtidos nesse trabalho são uma referência atual da teia de animais que co-ocorre com $P$. nattereri no lago Piranha um ambiente aquático saudável na Amazônia central

\section{Agradecimentos}

Agradecemos a Coordenação de Aperfeiçoamento de Pessoal de Nível Superior (CAPES) e ao Instituto Nacional de Pesquisas da Amazônia (INPA) pelo apoio financeiro, e a toda equipe do Laboratório de Parasitologia e Patologia de Peixes pelo auxílio técnico dado durante o estudo.

\section{Referências Bibliográficas}

AMATO, J.F.R., BOEGER, W.A. \& AMATO, S.B. 1991. Protocolos para laboratório: coleta e processamento de parasitos de pescado. Imprensa Universitária, Rio de Janeiro.

AYRES, M., AYRES, M.J.R., AYRES, D.L. \& SANTOS, A.A.S. 2003. BioEstat 3.0: aplicações estatísticas nas áreas de Ciências Biológicas e Médicas. Sociedade Civil Mamirauá, Belém.

BOEGER, W.A. \& THATCHER, V.E. 1988. Rhinergasilus piranhus gen. et sp. nov. (Copepoda: Poecilostomatoida: Ergasilidae) from the nasal cavities of piranha caju, Serrasalmus nattereri, in the central Amazon. P. Helm. Soc. Wash. 55(1):87-90. 
BRASIL-SATO, M.C. 1999. Ecologia das comunidades de parasitos metazoários de Pimelodus maculatus Lacépède, 1803 (Siluriformes: Pimelodidae) das bacias do rio São Francisco, Três Marias, MG e do rio Paraná. Tese de doutorado, Universidade Federal de São Carlos, São Paulo.

BUSH, A.O., LAFFERTY, K.D., LOTZ, J.M. \& SHOSTAK, A.W. 1997. Parasitology meets ecology on its own terms: Margolis et al. 1982, revisited. J. Parasitol. 83(4):575-583.

CAMPOS, C.F.M. 2006. Fauna parasitária e alterações teciduais em duas espécies de peixes dos rios Aquidauana e Miranda, pantanal Sul MatoGrossense. Tese de Doutorado, Universidade Estadual Paulista Júlio de Mesquita Filho, Botucatu, São Paulo.

CARVALHO, L.N., ARRUDA, R. \& DEL-CLARO, K. 2004. Host-parasite interactions between the piranha Pygocentrus nattereri (Characiformes: Characidae) and isopods and branchiurans (Crustaceae) in the rio Araguaia basin, Brazil. Neo. Ichthyol. 2(2):93-98.

CHUBB, J.C. 1973. Influence of parasites on freshwater fishes in Britain. Verh. Internat. Verein. Limnol. 18: 1628-1632.

DIAS, P.G., FURUYA, W.M., PAVANELLI, G.C., MACHADO, M.H., \& TAKEMOTO, R.M. 2004. Carga parasitária de Rondonia rondoni. Travassos 1920 (Nematoda, Atrictidae) e fator de condição do armado. Pterodoras granulosus, Valenciennes, 1833 (Pisces, Doradidae). Acta Scient. 26(2):151-156.

EIRAS, J.C., TAKEMOTO, R.M., \& PAVANELLI, G.C. 2006. Métodos de estudo e técnicas laboratoriais em parasitologia de peixes. 2. ed. Editora da Universidade Estadual de Maringá, Maringá, Paraná.

FINK, W.L. 1993. Revision of the piranha genus Pygocentrus (Teleostei, Characiformes). Copeia (3):665-687. doi:10.2307/1447228

FURTADO, J.I. \& TAN, K.L. 1973. Seasonal ocurrence of helminths in freshwater fishes. Part IV. Adult Cestoda, Nematoda and Acanthocephala. Adv. Parasitol. 20:1-292.

GUIDELLI, G.M. 2006. Comunidades parasitárias em espécies do gênero Leporinus de diferentes categorias tróficas e ambientes da planície de inundação do Alto rio Paraná. Tese de Doutorado. Universidade estadual de Maringá, Paraná.

ISAAC, A.,GUIDELLI, G.M., FRANÇA, J.G. \& PAVANELLI, G.C. 2004. Composição e estrutura das infracomunidades endoparasitárias de Gymnotus spp. (Pisces: Gymnotidae) do rio Baía, Mato Grosso do Sul, Brasil, Acta Scient. 26(4):453-462.

JUNK, W.J., BAYLEY, P.B. \& SPARKS, R.E. 1989. The flood pulse concept in river-floodplain systems. Can. J. Fish. Aquat. Sci. 106:110-127.

KADLEC, D., SIMKOVÁ, A., GELNAR, M. \& JARKOVSKY, J. 2003. Parasite communities of freshwater fishes under flood conditions. Parasitol. Res. 89:272-283.

LE CREN, E.D. 1951. The length-weight relationship and seasonal cycle in gonad weight and condition perch Perca fluviatilis. J. An. Ecol. 20:201219. doi: $10.2307 / 1540$
LEMLY, A. D. 1980. Effects of a larval parasite on the growth and survival of young bluegill. Proc. Ann. Conf. S. E. Assoc. Fish \& Wildl. Agencies. 34:263-274

LIZAMA, M.A.P. 2003. Estudo da relação entre a comunidade parasitária, meio ambiente e dinâmica da população de Prochilodus lineatus (Valenciennes, 1836) e Astyanax altiparanae Garutti \& Bristki, 2000, na planície de inundação do Alto rio Paraná, Brasil. Tese de Doutorado, Universidade Estadual de Maringá, Maringá, Paraná.

MACKENZIE, K., WILLIANS, H.H.; WILLIANS, B., MCVICAR, A.H. \& SIDDAL, R. 1995. Parasites as indicators of water quality and the potential use of helminth transmission in marine pollution studies. Adv. Parasitol. 35:85-144. doi:10.1016/S0065-308X(08)60070-6

MADI, R.R. 2005. Utilização dos helmintos parasitos de Geophagus brasiliensis (Quoy \& Galmard, 1824) (Cichlidae: Perciformes) como indicadores ambientais. Tese de Doutorado, Universidade estadual de Campinas, Campinas, São Paulo.

MALTA, J.C.O. 1994. Miracetyma piraya sp. nov. (Copepoda, Ergasildae) das brânquias de Pygocentrus nattereri (Kner, 1858) (Characiformes: Serrasalmidae) da Amazônia Brasileira. Acta Amazon. 23(2-3):261-269.

MALTA, J.C.O. \& VARELLA, A.M.B. 1996. Ergasilus yumaricus sp. n. (Copepoda: Ergasilidae) das brânquias de Pygocentrus nattereri (Kner, 1858), Serrasalmus rhombeus (Linnaeus, 1819) e Pristobrycon eigenmanni (Norman, 1929) (Characiformes: Serrasalmidae) da Amazônia Brasileira. Acta Amazon. 25(1-2):93-100.

MARCOGLIESE, D.J. \& CONE, D.K. 1997. Parasite communities as indicators of ecosystem stress. Parassitologia 39:227-232.

OVERSTREET, R.M. 1997. Parasitological data as monitors of environmental health. Parassitologia 39:169-175.

PAVANELLI, G.C., MACHADO, M.H. \& TAKEMOTO, R.M. 1997. Fauna helmíntica de peixes do rio Paraná, região de Porto Rico, Paraná. In A planície de inundação do alto rio Paraná: Aspectos físicos, biológicos e sócioeconômicos (A.E.A.M. Vazzoler, A.A. Agostinho \& N.S. Hahn, eds.). Editora da Universidade Estadual de Maringá, Maringá, p.307-329.

POULIN, R. 1992. Toxic pollution and parasitism freshwater fish. Parasitol. Today. 8:58-61. doi:10.1016/0169-4758(92)90090-O

SANTOS, G., FERREIRA, E. \& ZUANON, J. 2006. Peixes comerciais de Manaus. Ibama, Manaus.

SERRA-FREIRE, N.M. 2002. Planejamento e análise de pesquisas parasitológicas. Editora da Universidade Federal Fluminense, Niterói.

SURES, B. 2004. Environmental parasitology: relevancy of parasites in monitoring environmental pollution. Trends Parasitol. 20(4):170-177. PMid:15099556. doi:10.1016/j.pt.2004.01.014

TAKEMOTO, R.M., LIZAMA, M.A.P., GUIDELLI, G.M. \& PAVANELLI, G.C. 2004. Parasitos de peixes de águas continentais. In Sanidade de Organismos Aquáticos. (M.J.T. Ranzani-Paiva, R.M. Takemoto \& M.A.P. Lizama, eds.). Editora Varella, São Paulo, p.179-198.

ZAR, J.H. 1999. Biostatistical Analysis. $4^{\text {nd }}$ ed. Prentice Hall, New Jersey. 